\title{
Adiponectin: a pivotal role in the protection against cerebral ischemic injury
}

\author{
Ming-Hsiu Wu \\ Division of Neurology, Department of Internal Medicine, Chi Mei Medical Center, Liouying, Tainan 73657, Taiwan.
}

Correspondence to: Dr. Ming-Hsiu Wu, Division of Neurology, Department of Internal Medicine, Chi Mei Medical Center, Liouying, No.201, Taikang, Taikang Vil., Liuying Dist., Tainan City 736, Taiwan 73657, Taiwan. E-mail: galenmhwu@yahoo.com

How to cite this article: Wu MH. Adiponectin: a pivotal role in the protection against cerebral ischemic injury. Neuroimmuno/ Neuroinflammation 2019;6:4. http://dx.doi.org/10.20517/2347-8659.2019.07

Received: 20 Feb 2019 Accepted: 6 Mar 2019 Published: 22 Mar 2019

Science Editor: Athanassios P. Kyritsis Copy Editor: Cai-Hong Wang Production Editor: Huan-Liang Wu

Adiponectin (APN), an adipokine which weights $30 \mathrm{kDa}$, is first identified in 1995 and almost exclusively secreted by adipocytes ${ }^{[1]}$. Its physiological and clinical significance has been extensively explored in these years. A comprehensive review of adiponectin and its relating significance is beyond the scope of this article. Although its relationship with cerebral ischemia and ischemic stroke has been reviewed previously ${ }^{[2,3]}$, I try to briefly address the pivotal role of adiponectin in the protection against cerebral ischemic injury in the current article.

\section{STRUCTURE AND BIOSYNTHESIS}

There are 3 major oliogmeric multimers of APN: the low-molecular-weight trimer, the middle-molecularweight hexamer, and the high-molecular-weight (HMW) 12-18 multimers ${ }^{[4,5]}$. HMW adiponectin has been proposed to be the most potent form and drives the physiological role of adiponectin, as evidenced by some in vitro and human studies ${ }^{[1,6]}$. Also, a globular fragment of adiponectin (gAd) exists. It is generated as the full-length adiponectin is cleaved by leukocyte elastase which are secreted from activated monocytes and/or neutrophils. As compared with other isoforms, it remains at low circulating levels, only accounting for about $1 \%$ of total adiponectin ${ }^{[7]}$. The expression of adiponectin are under the regulation of several transcriptional factors, including CCAAT-enhancer-binding proteins, peroxisome proliferator-activated receptor $\gamma$, and sterol regulatory element binding protein $1 c^{[6]}$.

\section{PHYSIOLOGICAL AND PATHOPHYSIOLOGICAL ROLES IN HUMAN DISEASES}

The physiological role of adiponectin is mainly involved in insulin sensitivity and regulation of metabolism of glucose and lipids ${ }^{[8]}$. Furthermore, it has pleiotropic effects including anti-inflammation, anti-

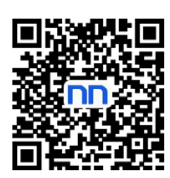


atherosclerosis, anti-thrombosis, and promotion of endothelial repair and angiogenesis which are protective in endothelial injury, atherosclerosis, and cardiovascular diseases ${ }^{[9-11]}$.

Adiponectin is abundant in peripheral circulation, representing about $0.01 \%$ total plasma proteins ${ }^{[12]}$, and has a rapid turnover rate ${ }^{[10]}$. Decreased circulating adiponectin level is noted in obesity for increased oxidative stress in accumulated fat ${ }^{[13,14]}$. Clinically, hypoadiponectinemia has been noted in various diseases, such as ischemic stroke ${ }^{[15,16]}$, coronary artery disease, insulin resistance and diabetes, hypertension, dyslipidemia, metabolic syndrome, hepatic steatosis and fibrosis, and cancer ${ }^{[17]}$. On the contrary, hyperadiponectinemia has been noted in congestive heart failure and chronic kidney disease ${ }^{[18,19]}$.

\section{ADIPONECTIN IS PROTECTIVE AGAINST CEREBRAL ISCHEMIC INJURY}

\section{Exogenous accumulation from the circulation but not endogenous production in damaged brain tissues after cerebral ischemic injury}

In many pre-clinical studies, adiponectin has been consistently shown to be protective against cerebral ischemic injury. However, the expression of adiponectin after cerebral ischemic injury is not endogenous in ischemic cerebral tissues but exogenous from the peripheral circulation.

There are studies exploring the expression profiles of adiponectin after middle cerebral artery occlusion $(\mathrm{MCAO})$ in mice and rats. The study by Yatomi et al. ${ }^{[20]}$ showed that, plasma adiponectin levels peaked soon at 1-3 h, decreased later, reached the nadir in $48 \mathrm{~h}$, and then returned to baseline gradually in the rat after MCAO. However, the expression pattern of adiponectin in ischemic cerebral hemisphere differed. Adiponectin showed higher levels in ischemic cerebral hemisphere than non-ischemic one during $72 \mathrm{~h}$ to 7 days after ischemia/reperfusion injury. Moreover, its expression was evident in endothelium only, not in neurons, glia, or macrophages. Finally, its expression in the endothelium of ischemic hemisphere seemed to be exogenous from the circulation but not endogenous from damaged cerebral hemispheres since there was no mRNA expression of adiponectin detected by reverse transcription polymerase chain reaction in these area. Another study by Shen et al. ${ }^{[21]}$ showed similar findings. They found that adiponectin started to rise $1 \mathrm{~h}$ in ischemic hemisphere after cerebral ischemia/reperfusion injury in mice, peaked in 3 days and lasted till 7 days. They found the expression of adiponectin occurred only in vascular endothelial cells but not in neurons or glial cells. Furthermore, they could not find the mRNA expression of adiponectin in ischemic cerebral hemisphere. Taken together, adiponectin accumulates in vascular endothelial cells instead of de novo generation in ischemic brain after cerebral ischemic injury.

\section{Adiponectin alleviates cerebral ischemic injury through multi-mechanisms}

Adiponectin is protective against cerebral ischemic injury and the mechanisms accounting for this are diverse. The study by Nishimura et al. ${ }^{[22]}$ reported that adiponectin exerted a cerebroprotective action through an endothelial nitric oxide synthase (eNOS)-dependent mechanism in cerebral ischemic injury. They showed that adenovirus-mediated delivery of adiponectin augmented the status of phosphorylation of endothelial nitric oxide synthase and reduced the infarction volume in adiponectin knockout (APN-KO) mice.

Another important mechanism of adipoenctin being protective against cerebral ischemic injury is antiinflammation. The study by Chen et al. ${ }^{[23]}$ showed that exogenous supplement of gAd via jugular vein reduced cerebral infarct size, neurological deficits, and expression of endogenous matrix metalloproteinase 9, interleukin (IL)-1 $\beta$, tumor necrosis factor- $\alpha$ and IL-8, and inhibited the translocation of nuclear factor (NF)$\kappa \mathrm{B}$ from cytoplasm into the nucleus in the rat after MCAO. The indirect evidence of its anti-inflammatory mechanism comes from another study by Jung et al. ${ }^{[2]}$. They found more rolling leukocyte and leukocyte adhesion were observed in the APN-KO mice than in the wide type mice after cerebral ischemia/reperfusion injury. They proposed that adiponectin inhibits the interaction between the endothelium and leukocytes and hence alleviates the inflammatory insult in cerebral ischemic injury. 
Adiponectin exerts anti-oxidation against cerebral ischemic injury as well ${ }^{[25,26]}$. The study by Song et al. ${ }^{[25]}$ showed that intracerebral injection of gAd attenuated infarct size and neurological deficits aggravated by NADPH oxidase activator in mice after MCAO along with increased activities of superoxide dismutase (SOD) and catalase, and reduced content of malondialdehyde (MDA). The study by Li et al. ${ }^{[26]}$ reported similar findings. They showed intraperitoneal supplement of adiponectin improved neurological deficits, decreased infarct size, and attenuated neuronal injury along with decreased MDA levels and increased SOD activity levels in mice after MCAO. The study by Wang et al. ${ }^{[27]}$ reported adiponectin attenuated oxygen and glucose deprivation-induced neuronal injury and mitochondrial oxidative stress in hippocampal neuronal HT22 cells as evidenced by attenuated reactive oxygen species and malondialdehyde, and increased superoxide dismutase and glutathione peroxidase activity.

Also, adiponectin has been related to $\mathrm{PKA}, \mathrm{CREB}$, and $\mathrm{BDNF}$ in the protection against cerebral ischemic injury. The study by Bai et al. ${ }^{[28]}$ reported activation of CAMP/PKA-CREB-BDNF signaling pathway by adiponectin was protective against ischemia/reperfusion injury with reduced infarct volume, neurological deficits and brain water content.

Finally, anti-apoptosis after cerebral ischemic injury by adiponectin has been found in in vivo and in vitro studies $^{[25-27,29]}$, including that by our group ${ }^{[30]}$.

\section{APN-gene modified cell therapy alleviates cerebral ischemic injury}

The pre-clinical studies of APN-gene modified cell therapies in the treatment of cerebral ischemic injury are growing recently. The study by Nishimura et al. ${ }^{[22]}$ showed that adenovirus-mediated expression of adiponectin reduced brain infarction volume, increased cerebral blood flow, and improved neurological deficits, through an eNOS-dependent mechanism in cerebral ischemic injury. The study by Shen et al. ${ }^{[31]}$ reported that adiponectin could promote focal angiogenesis in cerebral ischemic injury. They showed that after MCAO mice receiving intracerebral injection of adeno-associated viral vector carrying the APN gene had reduced ischemia-induced brain atrophy, improved neurological function and increased number of microvessels along with increased AMPK phosphorylation and vascular endothelial growth factor expression. Furthermore, the study by Miao et al ${ }^{[32]}$ showed this angiogenetic effect was more significant in aged mice than young mice.

Our group ${ }^{[30]}$ showed that pre-treatment of baculovirus-mediated expression of APN through intra-cerebral injection was protective against cerebral ischemic injury in both normal weight and obese rats through reducing brain infarct and edema, neurologic deficits, and p38-mediated neuronal apoptosis.

Recently, it has been shown that genetically-transplanted endothelial progenitor cells with adiponectin by lentivirus could reduce cerebral infarction volume, improve behavior outcome, increase microvessel density, and reduce cell apoptosis ${ }^{[33]}$.

\section{gAd alleviates cerebral ischemic injury}

Among adiponectin isoforms, the globular adiponectin has been mostly studied and consistently shown to be protective against cerebral ischemic injury ${ }^{[23,25,29]}$. The data regarding whether other isoforms of adiponectin could exert protection against cerebral ischemic injury are currently lacking and warrant further studies.

In summary, adiponectin, an adipokine secreted by adipocytes, exists in a relatively large amount in the peripheral circulation. During the cerebral ischemic injury, it accumulates in the damaged cerebral vasculature instead of de novo generation by damaged cerebral tissues. It exerts multiple protective mechanisms against cerebral ischemic injury including eNOS-dependent mechanism, anti-inflammation, anti-oxidation, anti-apoptosis, and promotion of angiogenesis. Since adiponectin is an adipokine naturally 
secreted by human adipose tissues, and has multi-mechanisms protective against cerebral ischemic injury, it is of great potential in the application of clinical treatment of ischemic stroke in the future.

\section{DECLARATIONS}

\section{Acknowledgments}

The author is grateful to Prof. Mao-Tsun Lin, Prof. Chao-Ching Huang, and Prof. Chun Y. Hsu for mentoring, and colleagues of Chi Mei Medical Center, and team members of Taiwan Stroke Biosignature for support.

\section{Authors' contributions}

$\mathrm{Wu} \mathrm{MH}$ is responsible for design and conceptualization of the study, drafting and revising the manuscript, and obtaining funding.

\section{Availability of data and materials}

Not applicable.

\section{Financial support and sponsorship}

This study was supported by grants from Chi Mei Medical Center (CMNCKU9908), Chi Mei Medical Center, Liouying (CLFHR10607), and Academia Sinica Taiwan Stroke Biosignature Project (AS-BD-108-11).

\section{Conflicts of interest}

The author declared that there are no conflicts of interest.

\section{Ethical approval and consent to participate}

Not applicable.

\section{Consent for publication}

Not applicable.

\section{Copyright}

(c) The Author(s) 2019.

\section{REFERENCES}

1. Yamauchi T, Kadowaki T. Physiological and pathophysiological roles of adiponectin and adiponectin receptors in the integrated regulation of metabolic and cardiovascular diseases. Int J Obes (Lond) 2008;32:S13-8.

2. Wu MH. Obesity, adiponectin and prognosis of ischemic stroke from bench to beside - basic and clinical studies. Taiwan: National Cheng Kung University; 2017. p. 111.

3. Bloemer J, Pinky PD, Govindarajulu M, Hong H, Judd R, et al. Role of adiponectin in central nervous system disorders. Neural Plast 2018;2018:4593530.

4. Pajvani UB, Du X, Combs TP, Berg AH, Rajala MW, et al. Structure-function studies of the adipocyte-secreted hormone Acrp30/ adiponectin. Implications fpr metabolic regulation and bioactivity. J Biol Chem 2003;278:9073-85.

5. Waki H, Yamauchi T, Kamon J, Ito Y, Uchida S, et al. Impaired multimerization of human adiponectin mutants associated with diabetes. Molecular structure and multimer formation of adiponectin. J Biol Chem 2003;278:40352-63.

6. Yamauchi T, Kadowaki T. Adiponectin receptor as a key player in healthy longevity and obesity-related diseases. Cell Metab 2013;17:185-96.

7. Waki H, Yamauchi T, Kamon J, Kita S, Ito Y, et al. Generation of globular fragment of adiponectin by leukocyte elastase secreted by monocytic cell line THP-1. Endocrinology 2005;146:790-6.

8. Guerre-Millo M. Adiponectin: an update. Diabetes Metab 2008;34:12-8.

9. Zhu W, Cheng KK, Vanhoutte PM, Lam KS, Xu A. Vascular effects of adiponectin: molecular mechanisms and potential therapeutic intervention. Clin Sci (Lond) 2008;114:361-74.

10. Caselli C, D'Amico A, Cabiati M, Prescimone T, Del Ry S, et al. Back to the heart: the protective role of adiponectin. Pharmacol Res 2014;82:9-20.

11. Hui X, Lam KS, Vanhoutte PM, Xu A. Adiponectin and cardiovascular health: an update. Br J Pharmacol 2012;165:574-90.

12. Trujillo ME, Scherer PE. Adiponectin--journey from an adipocyte secretory protein to biomarker of the metabolic syndrome. J Intern 
Med 2005;257:167-75.

13. Furukawa S, Fujita T, Shimabukuro M, Iwaki M, Yamada Y, et al. Increased oxidative stress in obesity and its impact on metabolic syndrome. J Clin Invest 2004;114:1752-61.

14. Matsuda M, Shimomura I. Roles of adiponectin and oxidative stress in obesity-associated metabolic and cardiovascular diseases. Rev Endocr Metab Disord 2014;15:1-10.

15. Chen MP, Tsai JC, Chung FM, Yang SS, Hsing LL, et al. Hypoadiponectinemia is associated with ischemic cerebrovascular disease. Arterioscler Thromb Vasc Biol 2005;25:821-6.

16. Marousi S, Theodorou G, Karakantza M, Papathanasopoulos P, Ellul J. Serum adiponectin acutely after an ischemic stroke: implications for a long-lasting, suppressed anti-inflammatory role. Acta Neurol Scand 2010;121:277-84.

17. Hossain MM, Mukheem A, Kamarul T. The prevention and treatment of hypoadiponectinemia-associated human diseases by upregulation of plasma adiponectin. Life Sci 2015;135:55-67.

18. Funahashi T, Matsuzawa Y. Adiponectin and the cardiometabolic syndrome: an epidemiological perspective. Best Pract Res Clin Endocrinol Metab 2014;28:93-106.

19. Nigro E, Scudiero O, Monaco ML, Palmieri A, Mazzarella G, et al. New insight into adiponectin role in obesity and obesity-related diseases. Biomed Res Int 2014;2014:658913.

20. Yatomi K, Miyamoto N, Komine-Kobayashi M, Liu M, Oishi H, et al. Pathophysiological dual action of adiponectin after transient focal ischemia in mouse brain. Brain Res 2009;1297:169-76.

21. Shen LH, Miao J, Zhao YJ, Zhao YJ, Liang W. Expression of brain adiponectin in a murine model of transient cerebral ischemia. Int J Clin Exp Med 2014;7:4590-6.

22. Nishimura M, Izumiya Y, Higuchi A, Shibata R, Qiu J, et al. Adiponectin prevents cerebral ischemic injury through endothelial nitric oxide synthase dependent mechanisms. Circulation 2008;117:216-23.

23. Chen B, Liao WQ, Xu N, Xu H, Wen JY, et al. Adiponectin protects against cerebral ischemia-reperfusion injury through antiinflammatory action. Brain Res 2009;1273:129-37.

24. Jung YS, Ha SK, Kim SD, Kim SH, Lim DJ, et al. The role of adiponectin in secondary inflammatory reaction in cerebral ischemia. J Cerebrovasc Endovasc Neurosurg 2013;15:171-6.

25. Song W, Huo T, Guo F, Wang H, Wei H, et al. Globular adiponectin elicits neuroprotection by inhibiting NADPH oxidase-mediated oxidative damage in ischemic stroke. Neuroscience 2013;248:136-44.

26. Li X, Guo H, Zhao L, Wang B, Liu H, et al. Adiponectin attenuates NADPH oxidase-mediated oxidative stress and neuronal damage induced by cerebral ischemia-reperfusion injury. Biochim Biophys Acta Mol Basis Dis 2017;1863:3265-76.

27. Wang B, Guo H, Li X, Yue L, Liu H, et al. Adiponectin attenuates oxygen-glucose deprivation-induced mitochondrial oxidative injury and apoptosis in hippocampal HT22 cells via the JAK2/STAT3 pathway. Cell Transplant 2018;27:1731-43.

28. Bai H, Zhao L, Liu H, Guo H, Guo W, et al. Adiponectin confers neuroprotection against cerebral ischemia-reperfusion injury through activating the cAMP/PKA-CREB-BDNF signaling. Brain Res Bull 2018;143:145-54.

29. Song W, Guo F, Zhong H, Liu L, Yang R, et al. Therapeutic window of globular adiponectin against cerebral ischemia in diabetic mice: the role of dynamic alteration of adiponectin/adiponectin receptor expression. Sci Rep 2015;5:17310.

30. Wu MH, Chio CC, Tsai KJ, Chang CP, Lin NK, et al. Obesity exacerbates rat cerebral ischemic injury through enhancing ischemic adiponectin-containing neuronal apoptosis. Mol Neurobiol 2016;53:3702-13.

31. Shen L, Miao J, Yuan F, Zhao Y, Tang Y, et al. Overexpression of adiponectin promotes focal angiogenesis in the mouse brain following middle cerebral artery occlusion. Gene Ther 2013;20:93-101.

32. Miao J, Shen LH, Tang YH, Wang YT, Tao MX, et al. Overexpression of adiponectin improves neurobehavioral outcomes after focal cerebral ischemia in aged mice. CNS Neurosci Ther 2013;19:969-77.

33. Zhang R, Xie X. Constitutive expression of adiponectin in endothelial progenitor cells protects a rat model of cerebral ischemia. Neural Plast 2017;2017:6809745. 